\title{
вм Global Health Drivers of antibiotic use in Vietnam: implications for designing community interventions
}

\author{
Shannon McKinn (D) , ${ }^{1}$ Duy Hoang Trinh, ${ }^{2}$ Dorothy Drabarek, ${ }^{1}$ Thao Thu Trieu, ${ }^{2}$ \\ Phuong Thi Lan Nguyen, ${ }^{3}$ Thai Hung Cao, ${ }^{4}$ Anh Duc Dang, ${ }^{3}$ Thu Anh Nguyen, ${ }^{1,2}$ \\ Greg J Fox, ${ }^{1,2}$ Sarah Bernays (i) ${ }^{1,5}$
}

To cite: McKinn S, Trinh DH, Drabarek D, et al. Drivers of antibiotic use in Vietnam: implications for designing community interventions. BMJ Global Health

2021;6:e005875. doi:10.1136/ bmjgh-2021-005875

Handling editor Seye Abimbola

Received 31 March 2021 Accepted 29 June 2021

\section{Check for updates}

(c) Author(s) (or their employer(s)) 2021. Re-use permitted under CC BY-NC. No commercial re-use. See rights and permissions. Published by BMJ.

${ }^{1}$ Faculty of Medicine and Health, The University of Sydney, Sydney, New South Wales, Australia

${ }^{2}$ Woolcock Institute of Medical Research, Glebe, New South Wales, Australia

${ }^{3}$ National Insitute of Hygiene and Epidemiology, Ministry of Health, Hanoi, Vietnam

${ }^{4}$ Medical Service Administration, Ministry of Health, Hanoi, Vietnam

${ }^{5}$ Public Health and Policy, London School of Hygiene and Tropical Medicine, London, UK

Correspondence to

Dr Sarah Bernays;

sarah.bernays@sydney.edu.au

\section{ABSTRACT}

Introduction Antimicrobial resistance is a global challenge that threatens our ability to prevent and treat common infectious diseases. Vietnam is affected by high rates of antimicrobial resistant infections, driven by the overuse of antibiotics and the Vietnamese government has recognised antimicrobial resistance as a health security priority. This study aimed to understand how people in Vietnam use antibiotics in community settings, and the factors that impact their practices and decisionmaking regarding antibiotics.

Methods We conducted 43 qualitative in-depth interviews with 50 community members in two urban and two rural sites in Vietnam. We conducted iterative, inductive thematic analysis alongside data collection through a process of systematic debriefings based on detailed interview summaries. Through this process, we developed a coding framework that was then applied to transcribed interview data.

Results Frequent and indiscriminate use of antibiotics was driven by the powerful appeal that antibiotics held for many Vietnamese consumers. Consumers were discerning in making decisions in their purchase and use of antibiotics. Consumers' decisions were affected by perceptions of what constitutes high-quality medicine (effective, strong, accessible and affordable); privileging symptom control over diagnosis; social constructions of antibiotics as a trusted remedy with embodied evidence of prior efficacy, which is reinforced by advice from trusted sources in their community; and varied, generally incomplete, understanding of the concept of antibiotic resistance and its implications for individuals and for public health.

Conclusion Antibiotic use at the community and primary care level in Vietnam is driven by community members' social and economic response to what constitutes effective healthcare, rather than biomedica logic. Community-based interventions to reduce unnecessary antibiotic use need to engage with the entangled socio-structural factors that 'resist' current public health efforts to ration antibiotic use, alongside biomedical drivers. This study has informed the design of a community-based trial to reduce unnecessary antibiotic use.

\section{Key questions}

What is already known?

- Antimicrobial resistance is a global public health threat.

- Vietnam is affected by high rates of antimicrobial resistant disease, partly driven by unnecessary antibiotic use.

- Approaches to reducing antibiotic use that focus on individual behaviour change have been largely unsuccessful in reducing unnecessary antibiotic consumption.

What are the new findings?

- Vietnamese consumers' decisions were affected by community perceptions of what constitutes highquality medicine; privileging symptom control over diagnosis; social constructions of antibiotics as a trusted remedy; and limited understanding of the concept of antibiotic resistance.

- Social networks play an important role in influencing and reinforcing consumers' purchase and consumption of antibiotics.

- The risks of antimicrobial resistance have been interpreted by community members as the risk of the individual being harmed by antibiotics, or becoming immune to antibiotics, rather than being understood as a risk to the community as a whole.

What do the new findings imply?

- Community-based interventions to reduce unnecessary antibiotic use need to engage with community values and structural conditions that drive antibiotic use, alongside biomedical drivers.

- Rather than simply discouraging antibiotic use, interventions should promote alternatives that provide similar perceived benefits as antibiotics.

\section{INTRODUCTION}

Antimicrobial resistance (AMR) is a global challenge that threatens our ability to treat common infectious diseases with antimicrobials. Drug-resistant disease causes over 700000 deaths worldwide per year, ${ }^{1}$ and is a considerable economic burden to health 
systems. ${ }^{1-4}$ Within 30 years, AMR is predicted to be among the leading causes of mortality globally, disproportionately impacting populations in low/middle-income countries (LMICs).

Vietnam is already experiencing high rates of antimicrobial resistant disease. Overuse of antimicrobials, of which antibiotics are a subset, is one of several factors (including poor quality medications, inadequate AMR surveillance, poor community awareness, inadequate regulation, excessive agricultural use and economic drivers) linked to rising rates of antimicrobial resistant infections in Vietnam. ${ }^{67}$ At the hospital level, approximately one-third of antibiotics used annually in Vietnam are thought to be taken unnecessarily, ${ }^{8}$ and this proportion is likely to be even higher at the community and primary care level, facilitated through private pharmacies and informal sellers servicing local markets. ${ }^{8}$

The Vietnamese health system has four levels: central, provincial, district and commune, with primary health services accessible to most of the population at the commune and district levels. However, there is an unofficial 'fifth level', with private pharmacies and drug outlets often used as the first point of primary care. ${ }^{910}$ This could be due to a range of factors, including their convenient opening hours, availability of common medicines, the ability to purchase medicines in small quantities, their geographical accessibility ${ }^{11}$ and their abundance. In 2015, there were an estimated 66 registered drug outlets per 100000 population in Vietnam; this figure does not include the significant number of unregistered drug outlets. The number of pharmacists is considerably lower, at 33.9 pharmacists per 100000 population, ${ }^{12}$ indicating that many outlets are manned by staff with minimal formal pharmacy training.

Unregulated sales of prescription medication are driven by high perceived, and actual, consumer demand for antibiotics. ${ }^{10} 13$ Despite laws necessitating the use of prescriptions, ${ }^{14}$ antibiotics are widely sold without prescription at private pharmacies and drug stores. ${ }^{10}{ }^{13}$ While the proportion of antibiotics sold without a prescription may be falling (estimations range from $88 \%$ to $91 \%$ in $2010^{13}$ compared with $58 \%$ in $2018^{15}$ ), non-prescription purchases still constitute the majority of antibiotic purchases from pharmacies. ${ }^{15}$ In previous studies, the majority of non-prescription purchases were for customers with symptoms of respiratory tract infections (cough, runny nose, sore throat) that are frequently viral, ${ }^{15}$ and fever. The antibiotics most commonly purchased over the counter were extendedspectrum penicillins such as amoxicillin and ampicillin, and first-generation cephalosporins, such as cephalexin. ${ }^{1315}$ In one study, around $40 \%$ of customers were provided with antibiotics classified by the WHO as antibiotics with higher resistance potential. ${ }^{15}$

The Vietnamese government has recognised AMR as a health security priority. ${ }^{16}$ However, to date, regulations to address inappropriate use and provision of antimicrobials have had limited impact. There is limited enforcement of legislation in practice, ${ }^{61315}$ as well as limited provisions in place for dealing with the consequences of enforcing such regulations, such as increased pressure on the public health system and economic losses to business owners. ${ }^{10}$ Interventions to reduce inappropriate use of antimicrobial have focused on hospital-level stewardship programmes, with little attention paid to mediating the large amount of antibiotics consumed at the community level. ${ }^{15}$

It has often been assumed that overprescribing and overdispensing of antimicrobials in LMICs is due to limited knowledge about AMR and antimicrobial use among prescribers and dispensers. ${ }^{17}$ However, evidence from LMICs demonstrates that while awareness of AMR is often high, awareness does not lead to reduced prescribing or dispensing of antimicrobials. ${ }^{18}$ Among pharmacy employees in Vietnam, knowledge of antibiotics and AMR is mixed. Previous studies show a wide gap between knowledge and practice of pharmacists. ${ }^{19-21}$ Among Vietnamese communities, knowledge of antibiotics and awareness of AMR have also been shown to be low, particularly in rural areas. ${ }^{132}$

While the problem of AMR is increasingly being recognised as requiring intersectoral (eg, agriculture, environment, health) and systemic interventions at national and regional levels, efforts to address consumption tend to be focused on the level of the individual. ${ }^{23} 24$ Approaches to reducing antibiotic use often focus on awareness raising, education and individual behaviour change (eg, changing prescribing practices), in an effort to improve knowledge which is assumed will lead to altered practices. However, recent research has shown that this focus on increasing knowledge and changing the behaviours of individuals has been largely unsuccessful in changing patterns of antibiotic consumption. Interventions which focus on behaviour change and individual responsibility and emphasise the biomedically appropriate use of antibiotics, while neglecting the powerful socio-structural drivers of antibiotic use, are inadequate to address the factors shaping the sale, demand and consumption of antibiotics. ${ }^{23}{ }^{25-28}$ Denyer Willis and Chandler argue that antibiotics function as a 'quick fix' for modern societies, conceptualising antibiotics, particularly in LMICs, as infrastructure that supports, or corrects for, weak systems of care, productivity and hygiene. ${ }^{25}$ To date, interventions to tackle AMR rarely engage with addressing substantial systemic weaknesses to engender necessary long-term changes in practice, focusing instead on the immediacy of individual behaviour change, which may be superficial and unsustainable: privileging short-term, measurable success at the expense of more complex, long-term structural solutions. ${ }^{25}$ Similarly, Broom $e t a l^{26}$ discuss the temporal myopia of modern life, a structural barrier which prevents the consideration of the long-term outcome of antimicrobial security in the face of the immediate demands of treating infection and achieving a positive patient outcome. 
Current research therefore indicates that interventions which are premised on providing accurate information that antibiotics are not appropriate to treat a range of viral infections will be insufficient to interrupt unnecessary antibiotic use. Instead, there needs to be a focus on the drivers of use within communities, which attends to the local context of consumption, and extends beyond presuming that biomedical logic is a primary concern in shaping or changing individuals' engagement in antibiotic use. This study aimed to understand how people in Vietnam use antibiotics, and the factors that impact their practices and decision-making around antibiotics. The findings and key recommendations of this qualitative study have been used to inform the design of community interventions (including health promotion materials, resources for pharmacists and communication strategies) to reduce unnecessary antibiotic use within the primary care level of the health system. These interventions are to be implemented in a community-based trial, the start of which is currently postponed due to COVID-19.

\section{METHODS}

\section{Study design}

This study is underpinned by an interpretivist theoretical perspective, a broad epistemological approach that is concerned with how individuals subjectively interpret or construct their experiences in relation to subjective and socially constructed contexts. We used a qualitative design to capture how individuals understand, interpret, and rationalise their own experiences with antibiotics, and the extent to which antibiotic use is shaped by multiple aspects of daily life.

\section{Setting}

We conducted interviews between April and September 2019 in two sites (one rural, one urban) in the municipality of Hanoi in the north of Vietnam, and two sites (one rural, one urban) in Ca Mau Province in the south. Sites were chosen in order to capture potential regional and cultural differences as well as urban/rural variation in the data.

\section{Recruitment and sampling}

Any community members (defined as residing in Hanoi municipality or Ca Mau Province) aged 18 years and over were eligible to participate. Different recruitment strategies were used in Hanoi and Ca Mau, according to the administrative requirements of the respective local authorities. In Hanoi, staff from local health services identified eligible participants according to the criteria provided by research staff and facilitated introductions to potential participants. In $\mathrm{Ca} \mathrm{Mau}$, recruitment was initially facilitated by local health authorities. However, with permission from local authorities, most participants in Ca Mau (approximately 80\%) were approached by the interviewer in the community and recruited directly. Three people who were approached declined to participate for personal reasons.
Participants who completed an interview received 100000 Vietnamese dong (approximately US\$4.3) as compensation for their time. All participants were provided with written participant information statements prior to the interview. This information was also explained verbally to participants prior to the interview. All participants provided written consent.

Initially, a convenience approach to sampling was adopted to access a wide range of people for maximum variation. As data collection progressed, a theoretical sampling approach was developed, underpinned by our emerging thematic framework, in order to recruit from specific demographic groups who were reported to be responsible for making medical decisions for their households, such as mothers of young children and elders.

\section{Participant sample}

Fifty community members participated in 43 interviews (table 1). While the majority of interviews were conducted one on one, on five occasions, participants were interviewed in pairs and on one occasion as a trio (eg, married couples, sisters, parent and adult child). In each instance, the participants requested to be interviewed alongside a close relation for their convenience (eg, to save participants' time if multiple family members were being interviewed) or comfort (eg, if an older participant wished to have their adult child present because they usually helped them with their health issues present). While multiple participant interviews can present issues for privacy and informed consent in some circumstances, particularly if unexpected and/or sensitive topics come to light, early interviews did not demonstrate that discussions around practices and beliefs around antibiotics elicited sensitive information, and the data obtained from multiparticipant interviews were not notably different from data obtained in one-on-one interviews. Each participant was only interviewed once. Participant occupations included small business owners, street vendors, motorbike taxi drivers, farmers, housewives/stay-at-home mothers, manufacturing workers, retired people and war veterans. Self-employment and informal sector work was common in both rural and urban study sites. In rural areas, this tended to be work related to agriculture and primary industries; in urban areas informal sector work was mostly related to small retail businesses and transport.

\section{Data collection}

In-depth interviews were conducted by local Vietnamese research staff (two women, one men) employed by the Woolcock Institute of Medical Research (DTH, TTT) and the National Institute of Hygiene Epidemiology (PTLN). All interviewers had previous experience conducting qualitative interviews and received further training from senior researchers prior to data collection, as well as ongoing support and supervision throughout the study. Interviewers did not have established relationships with participants prior to study commencement. Interviews were conducted in participants' homes, workplaces, 
Table 1 Participant characteristics

\begin{tabular}{|c|c|c|c|c|c|c|c|}
\hline \multirow[b]{2}{*}{ Characteristics } & \multicolumn{3}{|c|}{ Northern region } & \multicolumn{3}{|c|}{ Southern region } & \multirow[b]{2}{*}{ Total } \\
\hline & Rural & Urban & Total (\%) & Rural & Urban & Total (\%) & \\
\hline \multicolumn{8}{|l|}{ Gender } \\
\hline Female & 9 & 5 & $14(28)$ & 11 & 6 & $17(34)$ & $31(62)$ \\
\hline Male & 7 & 3 & $10(20)$ & 6 & 3 & $9(18)$ & $19(38)$ \\
\hline \multicolumn{8}{|l|}{ Age (range 25-90) } \\
\hline $25-34$ & 3 & 1 & $4(8)$ & 1 & 2 & $3(6)$ & 7 (14) \\
\hline $35-44$ & 2 & 1 & $3(6)$ & 4 & 1 & $5(10)$ & $8(16)$ \\
\hline $45-54$ & 5 & 1 & $6(12)$ & 1 & 2 & $3(6)$ & $9(18)$ \\
\hline $55-64$ & 2 & 2 & $4(8)$ & 3 & 2 & $5(10)$ & $9(18)$ \\
\hline $65-74$ & 0 & 3 & $3(6)$ & 7 & 2 & $9(18)$ & $12(24)$ \\
\hline$\geq 75$ & 4 & 0 & $4(8)$ & 1 & 1 & $2(4)$ & $6(12)$ \\
\hline \multicolumn{8}{|l|}{ Employment } \\
\hline Formal sector & 5 & 4 & $9(18)$ & 4 & 0 & $4(8)$ & $13(26)$ \\
\hline Informal sector & 4 & 1 & $5(10)$ & 6 & 5 & $11(22)$ & $16(32)$ \\
\hline Unpaid/dependent & 6 & 2 & $8(16)$ & 0 & 2 & $2(4)$ & $10(20)$ \\
\hline Pension & 1 & 1 & $2(4)$ & 7 & 2 & $9(18)$ & $11(22)$ \\
\hline Total & 16 & 8 & $24(48)$ & 17 & 9 & $26(52)$ & $50(100)$ \\
\hline
\end{tabular}

and other venues convenient to the participants such as coffee shops and health facilities.

Interviewers used a flexible topic guide covering key areas of investigation (box 1). The topic guide was continuously refined throughout the data collection period, as part of the iterative analysis process whereby initial analyses based on systematic debriefing discussions ${ }^{29}$ informed ongoing sample selection and areas of inquiry. Interviews lasted from 30 to $100 \mathrm{~min}$, with a median length of $60 \mathrm{~min}$. Interviews were audio recorded and transcribed in Vietnamese. Transcriptions were then translated to English. Detailed summaries, based on listening to the audio recordings and supplemented by fieldnotes, were written up as soon as was feasible, usually within 1 or 2 days of the interview. Transcripts were not returned to participants for checking. Data collection continued until thematic saturation was reached (ie, new data had become broadly repetitive of previously collected data in regard to the key

\section{Box 1 Interview topic guide}

\section{Key areas of investigation}

- Approaches to healing and treatment.

- Experiences of using antimicrobials.

- Access to antimicrobials.

- Attitudes to and knowledge of antimicrobials.

- Circulation, assimilation and rejection of information about health, medicine and antimicrobials in the community.

- Trusted and influential sources of health information in the community.

Future solutions to antimicrobial resistance. themes), ${ }^{30} 31$ which was determined through regular analytical meetings throughout the data collection period.

\section{Data analysis}

We conducted iterative, inductive (data-driven) thematic analysis alongside data collection. ${ }^{32}{ }^{33}$ Detailed written summaries formed the basis of systematic debriefing discussions (SM, DD, SB, DTH, TTT, PTLN) which were held via video call after every two to four interviews. These analytical discussions guided the identification of emerging themes and new areas of inquiry, as well as the recognition of saturation. ${ }^{29}$

Based on these extensive systematic debriefings and the detailed written interview summaries, SM developed the initial thematic coding framework. DTH, TTT and PTLN then each purposively selected a subset of transcripts to apply the coding framework, to check the comprehensiveness of the framework. Twenty-one transcripts were selected in order to achieve a broad variation of data within each subset. Analytical discussions were held regularly to update the coding framework and facilitate constant comparison across the team to ensure consistency. All transcripts were then coded in Microsoft Word, using the comment function. Coded and summarised data were organised and charted using Google Sheets, taking note of alternative perspectives and outliers and adding additional illustrative quotes to the spreadsheet of coded and summarised data. Themes were then developed by looking at relationships between codes and data extracts, before being reviewed, refined and discussed with all authors. ${ }^{32}$ 


\section{Patient and public involvement}

The public were not directly involved in designing or conducting this study. This study is a part of the larger V-RESIST Study (Combating the Emergence and Spread of Antimicrobial Resistant Infectious Diseases in Vietnam). The study established a stakeholder group which consists of a large-scale multidisciplinary collaboration between the health sector, academia and national policymakers from across Vietnam. This group meets biannually to work collaboratively on the design and development of interventions around AMR.

Participants have not provided feedback on the findings. Feedback to participants is being prepared through the community-based trial (including presentation of findings and how they have informed the design of intervention material and communication strategies to community stakeholders and elicitation of community feedback on these); however, the trial has been delayed due to COVID-19-related restrictions on public gatherings.

\section{RESULTS}

We found that antibiotics were used in the community for a variety of conditions and symptoms that often do not require antibiotics, including viral upper respiratory tract infections, pain, cuts and abrasions. Within the qualitative study, we are unable to make a judgement as to whether their own use of antibiotics was clinically 'appropriate'. However, participants reported that their decisions to purchase and use antibiotics for these conditions were generally made independently, or as recommended by pharmacy staff, not in consultation with a qualified prescribing clinician. Our findings demonstrate that the decision to use antibiotics can be at least partly explained by community perceptions of what constitutes a highquality medicine; privileging control of symptoms over receiving a diagnosis; and social constructions of antibiotics as a trusted remedy for a wide range of conditions. Some participants modified their use of antibiotics based on concerns about AMR. However, what AMR meant and how to effectively address it was widely misunderstood.

\section{The appeal of antibiotics}

Antibiotics were considered broadly synonymous with Western medicines, distinct from the oppositional category of 'traditional' medicines, and constituted a large proportion of Western medicines used by community members. Their appeal and credence were based on their perceived 'Western' origin, rather than on being antibiotics per se. Specifically, their appeal was predicated on their reputation for strength and their credibility was conferred by the tabular form and that they could be purchased in biomedical pharmacies. Antibiotics were emblematic of this broader category of 'Western' medicines, which were considered to indicate efficacy, strength, accessibility and affordability.
When I'm sick, I have to buy antibiotics. When I'm seriously sick, antibiotics will cure my sickness. (28 years old, female, rural, Hanoi)

Efficacy and strength were demonstrated through perceived rapid relief of symptoms, which allowed people to experience minimal disruption to their daily activities. It is important to note that many conditions for which Vietnamese people use antibiotics are self-limiting, and people are frequently provided with supplementary over-the-counter medication when they buy antibiotics. Improvements that may be due to the passage of time or non-antimicrobial over-the-counter medication are therefore attributed to antibiotics.

Community members commonly gave a time frame of 2-3 days as the expected time to recover from illness if using 'Western' medications, with one participant likening the rapidity of effect to 'instant noodles' (51 years old, male, urban, Ca Mau).

With Western medicines, people only need three days to recover and come back to work. (31 years old, female, rural, Hanoi)

Participants consistently characterised antibiotics as being inherently powerful, strong medications. Having partially absorbed messaging around the need for rationing such powerful medications, including potentially through a series of national AMR public health campaigns, many participants were concerned about antibiotics' potential for harm, even when using recommended regimens.

I don't take that medication too much because it has many harms. I don't know. People said that antibiotics make you feel tired. (81 years old, male, rural, Hanoi)

It was common for participants to discuss self-regulating their dosage and course of antibiotics, in order to limit exposure to potential toxicity and harm. However, rationing of antibiotics did not often translate to more targeted, less frequent use. Not taking medication 'too much' in the above quote refers to self-prescribing short, rather than infrequent, courses.

Some participants specifically mentioned this harmreduction approach to antibiotics in regard to their children, reflecting an underlying belief that balancing the strength and the potential toxicity of antibiotics was considered more risky for children. However, this was not a consistently found view, with other participants stating that their children required stronger medicine, as previously used medications had lost their effectiveness over time.

\section{Antibiotics as affordable, efficient healthcare}

Antibiotics were reported to be affordable and easily accessible. Despite regulations to the contrary, participants reported that it was common to purchase antibiotics over the counter at pharmacies and drug stores. Drug outlets are extremely common in the study area, with related research work showing that there were 133 
and 81.7 outlets per 100000 people in Hanoi municipality and Ca Mau Province, respectively (Beardsley et al 2021. Unpublished).

Pharmacies are all over the place. I can buy them at any store I want. It's convenient, your only job is to decide whether or not to buy them at this store. (66 years old, male, rural, Ca Mau)

Consumers exercised discretion regarding the quantity of medicines they bought, with single doses readily available, and frequently purchased by participants.

Participants reported that antibiotics reduced the cost of healthcare. The unit cost of tablets was mostly described as inexpensive but, more broadly, purchasing them was considered to be a cheap and convenient way to access what they considered to be effective and efficient healthcare. Participants even described purchasing antibiotics pre-emptively on a contingency basis:

No doctor's permission [prescription] needed. (...) People here often go to [Name]'s pharmacy and tell him to sell two ampi [ampicillin] tablets, and two tetra [tetracycline]

tablets, just in case. (68 years old, female, rural, Ca Mau)

A minority of participants living with significant financial constraints said that while the cost of antibiotics was one of the factors that shaped their behaviour, the per unit cost was minor in comparison with the costs of engaging with the primary healthcare system, which was perceived to be cumbersome, time-consuming, burdensome and potentially unsatisfactory. Pursuing a prescription through a consultation with a doctor was presumed to either produce the same result (the purchase of antibiotics) or potentially curtailed access to antibiotics by the enforcement of circumspect prescribing practices. Circumventing the primary healthcare system by purchasing antibiotics directly from willing pharmacists not only reduced direct costs of healthcare, but also the indirect costs that accompanied engaging in primary healthcare, which would involve taking time off work and the delayed alleviation of symptoms.

I myself know that I should go to the doctor when I'm ill but going to the doctor means trading time for work... It's a dilemma. (...) If I go to the doctor this morning, I won't have money for food this morning, meaning that my children won't have anything to eat (...) I have to find the quickest solution to the problem (...) I choose to go to the pharmacy to buy Western medicines because it's quicker... (44 years old, female, urban, Ca Mau)

Many participants, in both rural and urban sites, were precariously employed without access to sick leave. Paying for antibiotics offset other larger costs related to ill health, including lost income, both for the patient and often for an accompanying family member, and the financial and opportunity costs of engaging with primary care, including travel costs and inconvenience. These opportunity costs had the potential to be larger in rural areas, where there were few local alternatives to the communelevel health facility. In contrast, urban participants rarely engaged with commune health facilities, choosing instead to access higher level public services, or private practitioners. Participants in rural areas would often travel long distances to reach their preferred higher level facility, if they perceived that the better quality of care provided would be worth the effort. But this effort was best saved for serious symptoms or specialist services. That antibiotics were considered to best meet their needs when they experienced more minor symptoms of illness reflects how the appeal of antibiotics is entangled with the structural inaccessibility of the primary healthcare system.

\section{Symptom alleviation prioritised over diagnosis}

Knowledge about whether a specific medicine was an appropriate treatment or not mattered less than its perceived ability to 'cure' (ie, alleviate symptoms) the problem quickly. While participants frequently selfprescribed antibiotics, self-diagnosis of a condition related to their symptoms was less common. There was minimal priority given to diagnosing the cause of illness if symptoms were not considered alarming but commonplace; instead they focused on the management and amelioration of symptoms. When recalling clinical consultations, while health professionals may have offered them diagnoses, many participants did not remember them.

I only need him to dispense medicines for me, so I didn't ask why I had that condition. Maybe he wrote it on the paper he gave me. But he didn't ask me many questions. In general doctors don't ask many questions. (56 years old, female, rural, Ca Mau)

Many participants perceived there to be little qualitative difference between visiting a pharmacy and buying antibiotics over the counter and consulting a clinician to receive a prescription. While participants trusted the clinical expertise of health professionals, their practical engagement with such expertise often privileged pharmaceutical expertise which endorsed their own trust in specific medications and served to further justify their confidence in self-prescribing, without the need for it to be endorsed by a clinician who might offer an expert diagnosis of their condition. In an illustration of this, participants often mentioned that the credibility of health professionals and drug sellers rested on their ability to prescribe and dispense what the consumer considered to be 'good medicines' that alleviate symptoms quickly, rather than being wholly dependent on formal qualifications. A notable exception to this arose when a child was sick. The benefits of consulting a clinician were better appreciated in the context of childhood illness.

There is a seller in this area who has no medical training, but she can dispense very good medicines, people come to her a lot. Other younger pharmacists around here cannot compete with her. They are all losing [business] to her, she just practices with her own experience in dispensing drugs, she's very good, very fast. That lady is popular, her medicines take effect very quickly and that makes people recommend her. (66 years old, male, rural, Ca Mau) 


\section{Social production of antibiotics as trusted}

People's trust in antibiotics was affirmed through witnessed (direct or indirect) and embodied evidence of that medicine having worked on previous occasions. Participants' personal experience guided decisions about future treatments. If an antibiotic was perceived to have been effective for a relatively similar symptom or illness in the past, this evidence was presumed to be sufficient to justify its repeat purchase, ordinarily without it being considered necessary to check this with a qualified prescriber. What constituted embodied evidence need not be personal. It could be passed on from close relatives, observed within social networks, or affirmed through anecdotal evidence from other trusted sources such as health professionals, and also community members without any formal medical training or qualifications.

In the past, my father used to buy it [ampicillin] for me to treat headache, flu, coughing. So, when I have a headache, flu, coughing, runny nose, I'll buy it. (40 years old, female, rural, Ca Mau)

Informal circulation of health-related information within social networks played an important role in knowledge transfer, particularly in rural areas. These interactions often occurred in locations which are considered community hubs, such as markets, and social occasions such as parties.

Sometimes when we get together we all talk about health (...) we report back to each other who does their job well and [make] recommendations to each other. We discuss it in this neighbourhood. When our children and grandchildren get sick, they will comment on each health facility, and others listen. (68 years old, female, rural, Ca Mau)

The reliance on embodied evidence was critical to positioning antibiotics as a trusted emblem of quality healthcare, which was known to be easily accessible. This operated in a reinforcing cycle: participants purchased antibiotics for a wide spectrum of symptoms and conditions, anticipating that they would be an effective solution; credibility was conferred on those who dispensed them, which elevated the trustworthiness of community members who engaged in recommending and selling antibiotics, which in turn confirmed the reliability of the health information that they gave and justified further purchases.

Many people buy medicines there (...) so I trust that drugstore, their medicines are good (...) like with my child for example, when she had a cold or a fever or something, she recovered after one or two doses (...) That medicine is effective right away. (25 years old, female, rural, Ca Mau)

\section{Social responses to AMR}

Most participants had some level of awareness about AMR due to health promotion campaigns that have occurred in recent years. However, very few of them were able to accurately explain the concept of antibiotic resistance.
Despite their pervasive popularity, as previously noted, participants described actively rationing their use of antibiotics. This rationing was manifested by shortening the course. The rationale for this was that doing so would reduce the perceived risk of the individual in developing their own specific resistance, indicating community misperceptions about AMR.

It is said on TV that we should not abuse the antibiotic. (45 years old, male, rural, Hanoi)

The residual effects of such campaigns appeared to be heightened sensitivity to the dangers of 'too many antibiotics' taken at a particular time, rather than understanding the need to target the use of antibiotics to bacterial infections and therefore the pertinence of accurate diagnoses of conditions. Several participants directly referred to concerns about AMR when discussing their practices around using small amounts of antibiotics.

I heard from the TV that excess use of antibiotics will cause tolerance, but I take only a little, not much. I take it only once or twice every month or every two months. (51 years old, male, urban, Ca Mau)

The risks of AMR have been interpreted by many in the community as the risks of toxicity (from powerful, highquality antibiotics) and the risk of the individual (ie, not specific microorganisms) becoming immune or tolerant to the therapeutic effects of antibiotics. The message that unnecessary antibiotic use may result in antibiotics not working in the future has been interpreted at the individual level rather than being understood as a risk for the community as a whole.

My children are used to taking antibiotics. Cough drops and stuff like that don't work anymore. (...) They can only use antibiotics now. (...) they don't respond to mild medicines anymore. (31 years old, female, rural, Hanoi)

\section{DISCUSSION}

This study provides insights into consumption practices of antibiotics within particular communities in Vietnam. Antibiotic use was frequent and driven by the powerful appeal that Western medicines generally, and antibiotics specifically, held for the vast majority of community participants. This appeal ran counter to, or at least independent of, biomedical logic. Nonetheless, consumers demonstrated active discernment when making the decision to request and use antibiotics. Our sample was predominantly female for several reasons. Our initial findings pointed towards women being a particularly relevant group engaged in purchasing antibiotics, which pushed our sampling approach towards women. We also had some difficulty engaging men with the study topic, suggesting that decision-making about and purchasing of antibiotics is largely (although not exclusively) a female activity. While this was a multisite study, we did not find noteworthy differences between northern and southern regions, or urban and rural sites in terms of participants 
Table 2 Key recommendations

\section{Demand side interventions}

- Key recommendation (KR) 1: Engagement with community values and practices around antibiotics

- KR2: Increase awareness of existing direct pathways to primary healthcare and prescribers

- KR3: Investment in marketing appropriate alternatives to antibiotics, emphasising alternatives with qualities valued in antibiotics: efficacy, strength, affordability, accessibility (eg, paracetamol for fever and pain relief; oral rehydration salts for diarrhoea)

- KR4: Mass communication campaign engaging with influential figures and social networks at various levels (eg, national, regional, commune), involving community 'champions' who are considered influential to promote key messages.

\section{Supply side interventions}

KR5: Enforcement of regulatory legislation, including regulations requiring prescription-based dispensing and registration of pharmacies

- KR6: Implementation of health system reforms to improve affordability of primary care, aimed at reducing direct and indirect costs

- KR7: Incentivisation of improved access to qualified prescribers, for example, after-hours care in communitybased primary healthcare centres

- KR8: Incentivisation of promotion of appropriate alternatives to antibiotics to relieve the symptoms of common illnesses practices or attitudes around antibiotics. Our findings highlight how individual behaviour is intrinsically both a response to, and a reflection of, structural conditions. Attention to these principles must inform the design of community interventions if progress is to be made in influencing the reduction unnecessary antibiotic use in Vietnam and elsewhere (see table 2 for key recommendations). Additionally, it should be noted that a wide range of stakeholders, including clinicians, pharmacists and policymakers, should be consulted to inform such interventions. While this paper speaks to the community perspective, the perspectives of these groups have been captured through related studies under the larger V-RESIST programme of work, and will be published separately.

'Inappropriate' patient demand for antibiotics is often used to explain antibiotic overuse, ${ }^{25}$ and Vietnam is no exception. A previous study on antibiotic sales in Vietnamese pharmacies declares that 'overuse of antibiotics in the community is caused by people buying antibiotics after self-diagnosis or diagnosis by, often poorly trained, healthcare providers ${ }^{13}$ but there has been very little consideration of the factors that drive people to buy antibiotics, and to self-medicate. Our findings demonstrate that, whether clinically indicated or not, antibiotics are highly valued, serving an important function in symptom management and the perceived attainment of 'good health'. Our findings on the appeal of antibiotics within the community reflect this 'quick fix' as antibiotics offer a means to circumvent the need to navigate primary healthcare, saving patient-consumers' time and money. ${ }^{34}$

Purchasing self-prescribed antibiotics is a mechanism to avoid participation in the formal healthcare system while accessing affordable, rapid care, with minimal impact on productivity. This reveals the shift towards the pharmaceuticalisation of care, where antimicrobial medicines are used as an alternative form of 'healthcare' in lieu of clinical attentiveness. ${ }^{25}$ Broom et $a l^{26}$ posit that universal access to healthcare would address the systemic causes of $\mathrm{AMR}$, as it removes the pressure for 'quick fix' solutions, while also preventing health problems that may require antibiotics. Addressing AMR in the Vietnamese context is thus not simply about discouraging antibiotic use and enforcing regulatory legislation. Alternative pathways of healthcare must be offered. In Vietnam, this requires strengthening both the accessibility and the acceptability of the primary care model so that care, including appropriate (legal) prescribing, is available to all and engaging in it is made more appealing (table 2: KR2, KR6, KR7). We found that community members often preferred to use private pharmacies as a proxy for primary care because of the convenience, and the low opportunity costs compared with accessing primary care facilities. There are studies pointing towards patient dissatisfaction with primary care services in Vietnam, with complaints often centring around long waiting times, poor attitudes of staff and unsatisfactory facilities. ${ }^{35}$ While patients will often self-refer to higher level district and provincial services that they perceive to be of higher quality ${ }^{35}$ (even it means travelling further and spending more), ${ }^{36}$ for the minor ailments for which antibiotics are often sought, private pharmacies offer an attractive and convenient substitute for primary care. Our findings reflect that decision-making about purchasing antibiotics often falls to those in caring roles (ie, women). It is an important question, but was beyond the remit of this study, whether this gendered pattern persists into broader decisionmaking about whether or not to engage in the healthcare system beyond the initial purchase of antibiotics. If interventions are to encourage people to engage with the primary care system, a gendered approach to understanding the decision to engage with primary care would be constructive.

The knowledge deficit model of simply educating and raising awareness about AMR has been demonstrated to be problematic. This model typically does not engage with the non-biomedical and systemic drivers of health behaviours, ${ }^{25-2737}$ and can have unforeseen consequences when information is translated through the prism of local knowledge. This is demonstrated by community 
members' understanding of AMR, in which participants justified their decisions to cut short their course of medication based on their interpretation of how to mitigate the perceived risk of AMR. Stopping a course of antibiotics once symptoms subside is common practice throughout the world, ${ }^{10} 38$ and there is now evidence that suggests that directives to take the full course of antibiotics are ill-advised. ${ }^{39}$ Although cost has commonly been put forward as a foundational driver of prematurely ending a course of antibiotics, both in Vietnam and elsewhere, ${ }^{1340}$ our findings indicate that rather than being a cost-based decision, people have absorbed the message from Vietnamese AMR awareness campaigns that by taking less than the full course they will be able to successfully and safely use antibiotics again next time they are ill.

Similarly, educating someone about the appropriate treatment for a viral versus a bacterial infection may not translate into reduced use of antibiotics if, to them, illness is defined by symptoms, and not a diagnosis. Drawing on Whyte $e t a l$ s concept of the symbolic nature of medicines ${ }^{41}$ illness is concretised by rendering the body economically unproductive. Antibiotics are thus valued for their symbolic association with enabling individuals to sustain their engagement in daily economic and relational productivity, making diagnosis at least temporarily irrelevant. Community interventions need to engage with these concepts in order to hope to be effective in reducing clinically unnecessary antibiotic use. Such an intervention needs to position an alternative treatment and/or behaviour that embodies similar values as antibiotics and serves similar perceived beneficial function (table 2: KR3, KR8). At the same time, interventions to reduce antibiotic use must consider the barriers inherent in the suggested alternative pathways if they are to be successful. One possible barrier is community opposition; the targeted reduction of antibiotic use can be perceived by community members as a denial of care, dismissal of their concerns and a lowering of the standard of care. ${ }^{42} 43$ The prioritisation of symptom alleviation over a diagnosis may also be partly driven by the reportedly predominant communication style of health professionals in Vietnam. Previous research has found that health professionals do not often focus on eliciting detailed information from patients, and do not encourage patients to ask questions or display curiosity about their symptoms/condition. ${ }^{44}$ The communication style typically favoured in formal, hierarchical interactions, such as that between a doctor and patient is often didactic and intended to be passively received by the patient. ${ }^{45}$

Community interventions to reduce antibiotic use should use the vehicle of the social production of trust to change the message about what constitutes appropriate antibiotic use and the promotion of alternatives. At the local level, the community endorses antibiotics as trusted medicines. Antibiotics are continually re-endorsed through witnessed and embodied 'evidence' of their efficacy among community members. It is unclear whether there is a source of information that can compete with this social network endorsement. The public health establishment does not necessarily need to compete with social networks in this regard but engaging with how trust is produced is recommended, both for engaging the community in health promotion messages, and to foster trust in the health system. This is particularly pertinent in rural areas, where trusted health information was often conveyed by specific influential and respected community members during interactions that occurred in community hubs, which were better defined in rural communities compared with the more diffuse and diverse spaces for community interaction that are found in urban areas. For this reason, identifying and engaging 'community champions' to promote key messages around reducing antibiotic use could be particularly useful in rural communities (table 2: KR4).

\section{Strengths and limitations}

Strengths of this study include a heterogeneous sample, a rigorous data analysis process that drew on the multidisciplinary perspectives of the research team, and continual meaningful involvement of the local research team in all stages of the study. Limitations include that self-reported practice in interviews may differ from actual behaviour, and this may invite an element of social desirability bias. We attempted to minimise this by using a neutral interviewer and assuring participants about confidentiality. While the sample for this study was heterogeneous and geographically diverse within Vietnam, due to the nature of the qualitative approach wider generalisability outside of Vietnam may be limited. However, by providing thorough descriptions of the research contexts and methods, we have aimed to assist readers to determine the transferability of the findings to other contexts and participant groups, as is appropriate to the qualitative approach. This study limits the scope of the findings to antibiotic use in humans. We acknowledge that antibiotic use in food production (eg, preventive medication of livestock and over-the-counter availability of animal antibiotics) is an important part of the landscape of AMR in Vietnam that was not addressed in this study.

\section{CONCLUSION}

This study demonstrates that unnecessary antibiotic use at the community and primary care level in Vietnam is driven by a social and economic response to what constitutes effective healthcare, rather than biomedical logic. These findings that engage with the socio-structural factors that drive unnecessary antibiotic use are to be operationalised in an intervention to be trialled, but they are also principles that need to be considered more broadly in the fight against AMR by focusing on the sociocultural and economic drivers of AMR alongside biomedical drivers. We have made recommendations that could target behaviour at the level of consumers, healthcare providers and drug sellers. These include increasing awareness of existing primary care pathways, 
incentivising increased accessibility to prescribers, promoting alternatives to antibiotics that speak to the values that people prize in antibiotics and engaging with the social production of trust via influential community members. However, we recognise that this study also highlights additional structural changes that would need to accompany these individual behaviour changes at various levels in order to successfully combat AMR.

Acknowledgements The authors wish to thank the National Institute of Hygiene and Epidemiology, and Dr Susan Luu and Dr Momoe Takeuchi (WHO) for their support and assistance with this study. We also thank Dr Justin Beardsley for his assistance.

Contributors SM contributed to data analysis and interpretation, drafting and critically revising the manuscript. DTH, TTT and PTLN contributed to data collection, data analysis and interpretation, and critically revising the manuscript. DD contributed to study design, data analysis and interpretation, and critically revising the manuscript. TAN, TCH, ADD and GF contributed to study design, and critically revising the manuscript. SB contributed to study design, data analysis and interpretation, and critically revising the manuscript. All authors gave final approval of the submitted manuscript, and all authors are guarantors.

Funding Funding for this research was provided by the Australian Department of Foreign Affairs and Trade (NHMRC APP1153346), with support by the Indo-Pacific Centre for Health Security. GF was supported by a Career Development Fellowship (NHMRC APP1148372).

Competing interests None declared.

Patient and public involvement Patients and/or the public were not involved in the design, or conduct, or reporting, or dissemination plans of this research.

Patient consent for publication Not required.

Ethics approval Ethics approval was obtained in both Vietnam (National Institute of Hygiene and Epidemiology VN01057/IORG0008555) and Australia (University of Sydney 2018/912).

Provenance and peer review Not commissioned; externally peer reviewed.

Data availability statement Data are available upon request. Data underlying our findings cannot be made public for ethical reasons, as they contain information that could compromise the privacy and consent of research participants. Data requests may be sent to the corresponding author.

Open access This is an open access article distributed in accordance with the Creative Commons Attribution Non Commercial (CC BY-NC 4.0) license, which permits others to distribute, remix, adapt, build upon this work non-commercially, and license their derivative works on different terms, provided the original work is properly cited, appropriate credit is given, any changes made indicated, and the use is non-commercial. See: http://creativecommons.org/licenses/by-nc/4.0/.

\section{ORCID iDs}

Shannon McKinn http://orcid.org/0000-0001-6384-1745

Sarah Bernays http://orcid.org/0000-0001-7628-8408

\section{REFERENCES}

1 Interagency Coordination Group on Antimicrobial Resistance. No time to wait: securing the future from drug-resistant infections. Report to the Secretary-General of the United nations, 2019. Available: https://www.who.int/antimicrobial-resistance/interagencycoordination-group/IACG_final_report_EN.pdf

2 World Bank. Drug-Resistant infections: a threat to our economic future. Washington, DC World Bank; 2017. http://documents1. worldbank.org/curated/en/323311493396993758/pdf/final-report.pdf

3 The Review on Antimicrobial Resistance. Tackling drug-resistant infections globally: final report and recommendations. London The Review on Antimicrobial Resistance; 2016. https://amr-review.org/ sites/default/files/160525_Final\%20paper_with\%20cover.pdf

4 World Health Organization. Global action plan on antimicrobial resistance. Geneva World Health Organization; 2015. https://apps. who.int/iris/bitstream/handle/10665/193736/9789241509763_eng. pdf
5 Pokharel S, Raut S, Adhikari B. Tackling antimicrobial resistance in low-income and middle-income countries. BMJ Glob Health 2019;4:e002104.

6 Nguyen KV, Thi Do NT, Chandna A, et al. Antibiotic use and resistance in emerging economies: a situation analysis for Viet Nam. BMC Public Health 2013;13:1158.

7 Government of Vietnam. National action plan on combating drug resistance in the period from 2013 to 2020; 2013.

8 Thu TA, Rahman M, Coffin S, et al. Antibiotic use in Vietnamese hospitals: a multicenter point-prevalence study. Am J Infect Control 2012;40:840-4.

9 Hoa NB, Tiemersma EW, Sy DN, et al. Health-seeking behaviour among adults with prolonged cough in Vietnam. Trop Med Int Health 2011;16:1260-7.

10 Nguyen $\mathrm{HH}$, Ho DP, Vu TLH, et al. "I can make more from selling medicine when breaking the rules" - understanding the antibiotic supply network in a rural community in Viet Nam. BMC Public Health 2019;19:1560.

11 Miller R, Goodman C. Performance of retail pharmacies in low- and middle-income Asian settings: a systematic review. Health Policy Plan 2016;31:940-53.

12 World Health Organization (WHO). The global health Observatory. pharmacists per 10,000 population, 2020. Available: https:// www.who.int/data/gho/data/indicators/indicator-details/GHO/ pharmacists-(per-10-000-population)

13 Nga DTT, Chuc NTK, Hoa NP, et al. Antibiotic sales in rural and urban pharmacies in northern Vietnam: an observational study. BMC Pharmacol Toxicol 2014;15:6.

14 Government of Vietnam. Ministry of health decision no 1847/2003/ QD-BYT about regulation of drug prescribing and Selling prescription only; 2003.

15 Nguyen NV, Do NTT, Nguyen CTK, et al. Community-Level consumption of antibiotics according to the aware (access, Watch, reserve) classification in rural Vietnam. JAC Antimicrob Resist 2020;2:dlaa048.

16 World Health Organization. Joint external evaluation of IHR core capacities of Viet Nam. Geneva: World Health Organization, 2017.

17 Radyowijati A, Haak H. Improving antibiotic use in low-income countries: an overview of evidence on determinants. Soc Sci Med 2003;57:733-44.

18 Pearson M, Chandler C. Knowing antmicrobial resistance in practice: a multi-country qualitative study with human and animal healthcare professionals. Glob Health Action 2019;12:1599560.

19 Chuc NT, Larsson M, Falkenberg T, et al. Management of childhood acute respiratory infections at private pharmacies in Vietnam. Ann Pharmacother 2001;35:1283-8.

20 Larsson M, Binh NT, Tomson G, et al. Private pharmacy staff in Hanoi dispensing steroids - theory and practice. Pharm Pract 2006;4:60-7.

21 Hoa NQ, Thi Lan P, Phuc HD, et al. Antibiotic prescribing and dispensing for acute respiratory infections in children: effectiveness of a multi-faceted intervention for health-care providers in Vietnam. Glob Health Action 2017;10:1327638.

$22 \mathrm{Ha}$ TV, Nguyen AMT, Nguyen HST. Public awareness about antibiotic use and resistance among residents in highland areas of Vietnam. Biomed Res Int 2019;2019:9398536.

23 Chandler CIR. Current accounts of antimicrobial resistance: stabilisation, individualisation and antibiotics as infrastructure. Palgrave Commun 2019;5:53.

24 Price L, Gozdzielewska L, Young M, et al. Effectiveness of interventions to improve the public's antimicrobial resistance awareness and behaviours associated with prudent use of antimicrobials: a systematic review. J Antimicrob Chemother 2018;73:1464-78.

25 Denyer Willis L, Chandler C. Quick fix for care, productivity, hygiene and inequality: reframing the entrenched problem of antibiotic overuse. BMJ Glob Health 2019;4:e001590.

26 Broom A, Kenny K, Prainsack B, et al. Antimicrobial resistance as a problem of values? Views from three continents. Crit Public Health 2020;5:1-13.

27 Denyer Willis L, Chandler CIR. Anthropology's contribution to AMR control. AMR Control 2018:84-108 http://resistancecontrol.info/wpcontent/uploads/2018/05/104-08-chandler.pdf

28 Broom A, Doron A. Antimicrobial resistance, politics, and practice in India. Qual Health Res 2020:30:1684-96.

29 McMahon SA, Winch PJ. Systematic Debriefing after qualitative encounters: an essential analysis step in applied qualitative research. BMJ Glob Health 2018;3:e000837.

30 Morse JM. The significance of saturation. Qual Health Res 1995;5:147-9. 
31 Saunders B, Sim J, Kingstone T, et al. Saturation in qualitative research: exploring its conceptualization and operationalization. Qual Quant 2018;52:1893-907.

32 Braun V, Clarke V. Using thematic analysis in psychology. Qual Res Psychol 2006;3:77-101.

33 Ritchie J, Spencer L, O'Connor W. Carrying out qualitative analysis. In: Ritchie J, Lewis J, eds. Qualitative research practice: a guide for social science students and researchers. London: Sage, 2003: 219-62.

34 Monnais L. The colonial life of pharmaceuticals: medicines and modernity in Vietnam. Cambridge: Cambridge University Press 2019.

35 Quyen BTT, Ha NT, Van Minh H. Outpatient satisfaction with primary health care services in Vietnam: multilevel analysis results from the Vietnam health facilities assessment 2015. Health Psychol Open 2021;8:205510292110151.

36 McKinn S, Linh DT, Foster K, et al. A qualitative analysis of factors that influence Vietnamese ethnic minority women to seek materna health care. BMC Pregnancy Childbirth 2019;19:243.

37 Haenssgen MJ, Xayavong T, Charoenboon N, et al. The consequences of AMR education and awareness raising: outputs, outcomes, and behavioural impacts of an antibiotic-related educational activity in Lao PDR. Antibiotics 2018;7:95.

38 Irawati L, Alrasheedy AA, Hassali MA, et al. Low-Income community knowledge, attitudes and perceptions regarding antibiotics and antibiotic resistance in Jelutong district, Penang, Malaysia: a qualitative study. BMC Public Health 2019;19:1292.

39 Llewelyn MJ, Fitzpatrick JM, Darwin E, et al. The antibiotic course has had its day. BMJ 2017;358:j3418.

40 Matin MA, Khan WA, Karim MM, et al. What influences antibiotic sales in rural Bangladesh? A drug dispensers' perspective. J Pharm Policy Pract 2020;13:20.

41 Whyte SR, van der Geest S, Hardon A. Social lives of medicine. Cambridge: Cambridge University Press, 2002.

42 Lindenmeyer A, Redwood S, Griffith L, et al. Recent migrants' perspectives on antibiotic use and prescribing in primary care: a qualitative study. Br J Gen Pract 2016;66:e802-9.

43 Chandler CIR, Hutchinson E, Hutchinson C. Addressing antimicrobial resistance through social theory: an Anthropologically oriented report. London London School of Hygiene and Tropical Medicine; 2016. https://researchonline.Ishtm.ac.uk/id/eprint/3400500/1/ Addressing\%20Antimicrobial\%20Resistance\%20Through\% 20Social\%20Theory\%20GOLD\%20VoR.pdf

44 McKinn S, Duong TL, Foster K, et al. 'I do want to ask, but I can't speak': a qualitative study of ethnic minority women's experiences of communicating with primary health care professionals in remote, rural Vietnam. Int J Equity Health 2017;16:190.

45 Laverack G, Huy Dap D. Transforming information, education and communication in Vietnam. Health Educ 2003;103:363-9. 